\title{
A Camera Distortion Model Calibration Method Based on Fixed Points
}

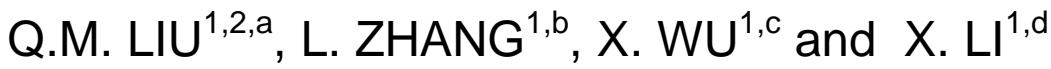 \\ ${ }^{1}$ College of Mechanical Engineering in Hangzhou Dianzi University, Hangzhou, Zhejiang, China \\ ${ }^{2}$ College of Information Engineering in Hangzhou Dianzi University, Hangzhou, Zhejiang, China \\ aqml@hdu.edu.cn, ${ }^{\mathrm{b}} 215821772 @ q q . c o m,{ }^{c} 1083133837 @ q q . c o m,{ }^{d}$ wx@163.com
}

Keywords: Pixel points, calibration board, automatic match, chessboard model

Abstract. A camera distortion model calibration method based on orientation points was proposed. This method needs to fix calibration plate on the measuring platform, and image sequences were obtained applying a calibration plate with five orientation points. The ellipse detection criteria are applied to eliminate the redundant information from images after pretreatment and all target points are determined as well. Target points are matched exactly from object space to image space. Camera parameters are solved and optimized by the Gauss-Newton. Experiments show that this method is more efficient, and calibration precision is higher.

\section{Introduction}

Digital close range photogrammetry is widely applied in mechanical manufacturing and electronic plating. Currently several methods of camera calibration have been proposed, such as traditional calibration method, self-correcting method and a tracking method based on active vision system ${ }^{[1]}$. Traditional calibration method uses block matching criterion to build constrained similarity relation. This method is simple operation, high precision to the calibration block and larger error ${ }^{[2]}$. Self-correcting method such as Zhang Zhengyou calibration method based on the chessboard mode. This modes is easy processing and have better robustness. Usually calibrations process needs high quality images to ensure accurate extraction of corner point. When calibration multiple images by this mode will suffere from the unsatisfied time consumption and maybe cause mismatch ${ }^{[3-4]} \mathrm{A}$ tracking method based on active vision system such as Faugeras proposed through the rotation of the camera to solve the parameter of the method. This method is only dealed with the first-order radial distortion $^{[5-6]}$.

Based on issues mentioned above. A new method for camera calibration was proposed. The method is in the premise of recognition and matching five orientation points, other target points are automatically matched from the target space to image space matched, then calculation and optimization camera parameters.

\section{The composition of the calibration system}

This paper system include: optical lens( TEC-M55 telephoto lens), image sensor(MER-150-50 digital camera), image acquisition card(IEEE1394), Image storage device, working platform, dot array calibration model, output control system and etl.as shown in Fig. 1.

Detection process: The dot array calibration model on measuring rotating platform fixation. In the one end of the rotating platform fixed camera. Through the camera collection gray image of the dot array calibration model. Then detection different location of the target point to determine the target images plane coordinates. Used for calibration process by the optical lens distortion correction of errors. Then through the transformation equation parameters to produce rotation Angle. Last matching with automatic calibration of camera parameters.

\section{Camera calibration method based on orientation point}

This paper proposes a new calibration plate based on the directional point, the ordinate origin of calibration plate is located in the calibration plate's the lower left corner. Columns of target points 
along the $\mathrm{X}$-axis positive direction were 11, and rows along the $\mathrm{Y}$-axis positive direction were 9, the total number of orientation points is 99 .

There were five major orientation points which uneven distribution(called target points), their diameter are obvious greater than other points. The diameter of large and small orientation point are $20 \mathrm{~mm}$ and $10 \mathrm{~mm}$ respectively, sign Orientation points 1 to 5 as follows. Spacing between two adjacent orientation points is $35 \mathrm{~mm}$. The calibration plate based on orientation points are shown in Fig. 2.

\section{Image acquisition and preprocessing}

The image distortion is associated with the distortion coefficient and the focal length of camera. In order to reduce the image quality and calibration results from the effects of camera distortions, Image Acquisition don't change the focal length. Calibration plate should be located at the center of the image position, it should be kept parallel to the camera and as far as possible.

In the process of camera calibration,target information needs to be preserved and additional background information should be removed.Firstly,to deal with the image using histogram modification and image filtering.Secondly,Application of Image Binarization Method for Image.After processing the results as shown in Fig. 3.
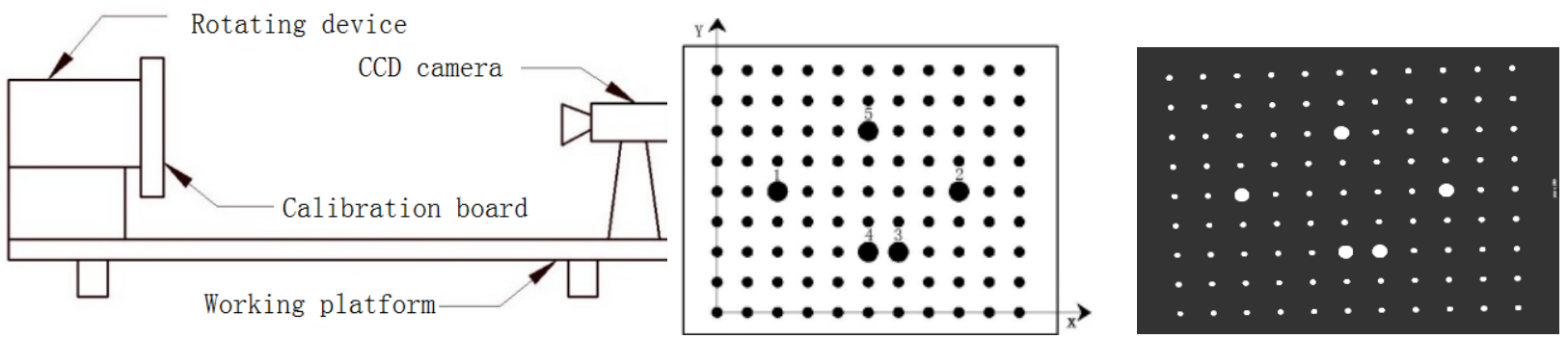

Fig. 1 working principle drawing

Fig. 2 The calibration plate Fig. 3 The results of image preprocessing

\section{Detection of target}

In the process of detecting target, need to target contour point information for further processing. This paper makes use of Canny algorithm for edge detection. High threshold set in 0.3, low threshold is set at 0.12.Fig.4 a interested area is extracted, The output is shown in Fig. 4(b).

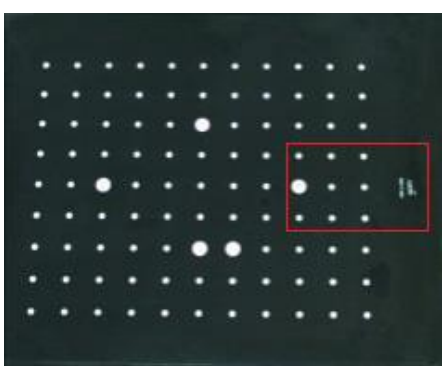

(a)the red zone

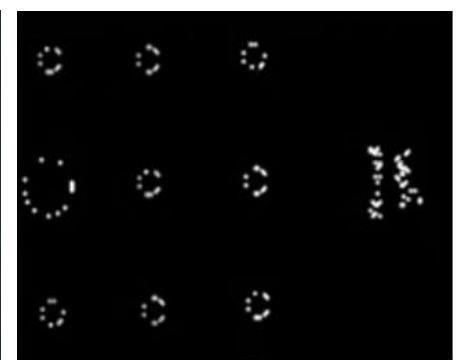

(b)Region of Interest Fig. 4 Enlarged edge detection

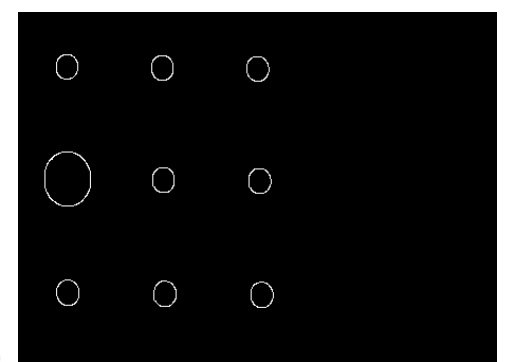

c. Part of the directional detection results

As we can see clearly from Fig. 4(a), there were other contours of impurities in the region of interest. By observing the interested region after the edge image, we found that the shape of the target from circular to elliptic. So this paper presents a ellipse detection standards to remove the redundant information of image and the ellipse fitting. Fig. 4(c) is the result of the Fig. 4(b) after the above steps, Finally only retain the elliptical orientation points and Superfluous information has been removed completely. 


\section{Automatic matching process}

Matching is designed to realize the pixel point and the target point corresponding relation. This algorithm is as follows:

(1)Looking for two longest ellipse in Fig.5, respectively to label them as F1 and F2, Find the two shortest distance of ellipse and named the F3 and F4.The remaining an ellipse, mark it as F5.

(2)looking for a farthest point from the F1 and F4 attachment sign Q1.In a similar way, find the farthest from the F1 and F5 attachment points in Q2.Calibration plate on the upper left corner and the lower left corner match to points P1 and P2.

(3)From left to right, find all parallel to the LP1P2, Let these lines pass all the points between LP1Q1andLP2Q2until the last line coincide with LQ1Q2.

Under the matlab software, mark points, which image point and target point are matched as red. Finally realizes the automatic matching. After the match result is shown in Fig. 6.

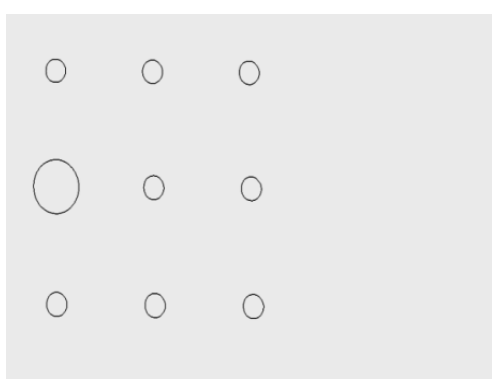

Fig. 5 The detecting results

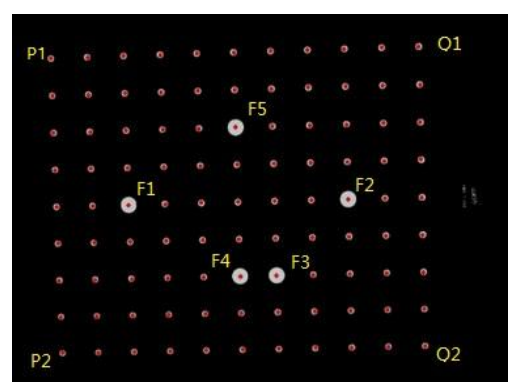

Fig. 6 The matching results

\section{Obtain calibration parameters}

After the completion of the match, The parameters of the camera are obtained by the following formula:

$$
\lambda\left[\begin{array}{c}
x \\
y \\
1
\end{array}\right]=\left[\begin{array}{c}
u \\
v \\
1
\end{array}\right]=K\left[\begin{array}{ll}
R & t
\end{array}\right]\left[\begin{array}{c}
X_{w} \\
Y_{w} \\
Z_{W} \\
1
\end{array}\right]=K\left[\begin{array}{llll}
r_{1} & r_{2} & r_{3} & t
\end{array}\right]\left[\begin{array}{c}
X_{w} \\
Y_{w} \\
Z_{W} \\
1
\end{array}\right]
$$

$\left(X_{w}, Y_{w}, Z_{w}\right)$ was the parameters in the world coordinate system, $(x, y)$ is the projection image coordinate values. $(u, v)$ is Projection value of the pixel coordinates midpoint. set $Z_{W}=0, R=\left[\begin{array}{lll}r_{1} & r_{2} & r_{3}\end{array}\right]$, The above formula can be converted into $K$ :

$$
K=\left[\begin{array}{ccc}
1 / d x & 0 & c_{x} \\
0 & 1 / d y & c_{y} \\
0 & 0 & 1
\end{array}\right]\left[\begin{array}{cccc}
f & 0 & c_{x} & 0 \\
0 & f & c_{y} & 0 \\
0 & 0 & 1 & 0
\end{array}\right]=\left[\begin{array}{cccc}
f_{x} & 0 & c_{x} & 0 \\
0 & f_{y} & c_{y} & 0 \\
0 & 0 & 1 & 0
\end{array}\right]
$$

Where, $d_{x}$ and $d_{y}$ was the actual size of a pixel in $x$ direction and $y$ direction of image plane. $f$ Is the focal length of the camera. $\left(c_{x}, c_{y}\right)$ is Camera center coordinates in the image coordinate system.

\section{Camera parameters optimization}

Because of a variety of distortion,we need to further optimize the parameters of the camera, This paper proposes a optimization method makes the minimum value: 
Solve the type involves complex nonlinear least squares problems, because in actual applications, the damping factor is difficult to determine, the problem such as slow convergence.This paper proposes a modified iterative algorithm.(1)all calibration parameters in (5) were represented by a vector $X$, then

(5) could be expressed as:

$$
\sum_{i=1}^{s}\left\|m_{i}-m(X)\right\|^{2}=\sum_{i=1}^{s} \sigma_{i}^{2}(X)
$$

(2)After iteration calculation, all vector of the objective function to find out.

$$
\sigma(X)^{(k)}=\left[\sigma_{1}\left(X^{K}\right), \sigma_{2}\left(X^{K}\right), \cdots, \sigma_{i}\left(X^{K}\right)\right]
$$

Standard parameter of the first order partial derivatives can be obtained from the type to solve:

$\alpha_{i j}=\frac{\partial \sigma_{i}\left(X^{(k)}\right)}{\partial X^{j}}(i=1,2 \ldots . . s ; j=1,2 \ldots n)$

The above formula can be expressed as a matrix:

$$
A_{k}=\left[\begin{array}{ccc}
\frac{\partial \sigma_{1}\left(X^{(k)}\right)}{\partial X^{1}} & \ldots & \frac{\partial \sigma_{1}\left(X^{(k)}\right)}{\partial X^{n}} \\
\vdots & \cdots & \vdots \\
\frac{\partial \sigma_{s}\left(X^{(k)}\right)}{\partial X^{1}} & \cdots & \frac{\partial \sigma_{s}\left(X^{(k)}\right)}{\partial X^{n}}
\end{array}\right]
$$

According to the maximum allowable error $\varepsilon$ and Maximum Iterations judge : if $\left\|X^{(k+1)}-X^{(k)}\right\| \leq \varepsilon$ or $k \geq \operatorname{Max}$, stop loop, And order $X=X^{(k+1)}$; otherwise $k=k+1$, Start cycle.The.camera, parameters .could. be, improved .more precisely .through,the above iteration.process.

\section{Experimental Verification and Analysis}

The following is taken from different angles by measuring the rotating platform to figure. 7 different azimuth calibration plate:

set $\varepsilon=10^{-9}, M a x=20$, The camera's internal parameters and distortion coefficient values as shown below:

$$
K=\left[\begin{array}{cccc}
1904.2321 & 0.0000 & 838.0323 & 0.0000 \\
0.0000 & 1905.1287 & 614.0071 & 0.0000 \\
0.0000 & 0.0000 & 1.0000 & 0.0000
\end{array}\right]
$$

Distortion coefficient $k_{1}=-0.084, k_{2}=0.14313$, in order to verify the robustness of new calibration method. The camera parameters $k^{\prime}$ using the improved checkerboard calibration method.

$$
K^{\prime}=\left[\begin{array}{cccc}
1947.5246 & 0.0000 & 819.1256 & 0.0000 \\
0.0000 & 1948.1482 & 598.0128 & 0.0000 \\
0.0000 & 0.0000 & 1.0000 & 0.0000
\end{array}\right]
$$


Comparing the results of $K$ and $\dot{K}$ As shown in table 1 from Table 1, A new calibration method relative to the board in the focal length and the center of the camera relative error in the values $\pm 2.5 \%$, Error in the can control range, In this paper, a new calibration method is verified by experiment with robustness.

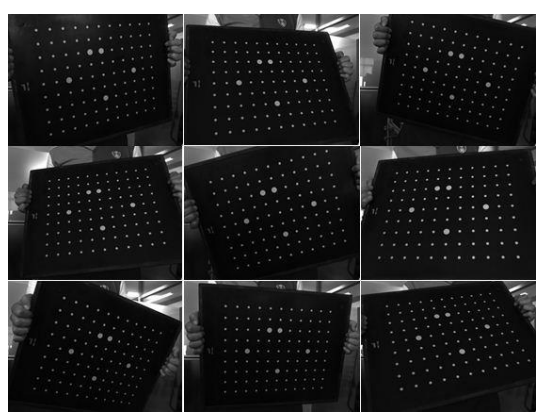

\begin{tabular}{lccc}
\multicolumn{4}{c}{ Table 1 camera parameters } \\
\hline $\begin{array}{c}\text { Parameter } \\
\text { s }\end{array}$ & $\begin{array}{c}\text { Chess board } \\
\text { calibration } \\
\text { method }\end{array}$ & $\begin{array}{c}\text { New calibration } \\
\text { method }\end{array}$ & relative error \\
\hline$f_{x}$ & 1947.4524 & 1904.6751 & $-2.250 \%$ \\
$f_{y}$ & 1948.5641 & 1905.1365 & $-2.2208 \%$ \\
$c_{x}$ & 819.1564 & 838.0456 & $2.3082 \%$ \\
$c_{y}$ & 598.5652 & 614.4561 & $2.6746 \%$ \\
\hline
\end{tabular}

Fig. 7 The results of image preprocessing

Projection error distribution can reflect the accuracy of camera calibration results.Distribution of the more densely populated, the more accurate calibration parameters. So the loop calibration mode and the new calibration model are proposed in this paper USES the same camera projection analysis, 9 image projection error as shown in Fig . 8.

In Fig. 8, in the direction of the error of the roughly 0.6 pixels, in the direction of the error of about 0.6 pixels

In the Fig. $8 \mathrm{~B}$, roughly 0.3 pixels, in the direction of the error in the direction of the error is only about 0.4 pixels.

In the Fig. $8 \mathrm{~B}$ projection error distribution is more dense.These results show that based on the camera calibration model of directional point than calibration cycle model based on a higher image quality is better.

Fig. 9 shows the new method and the traditional calibration method on the calibration time contrast, Calibration method based on directional point about $40 \mathrm{~s}$, take the traditional calibration approach takes about $70 \mathrm{~s}$, the results show that the proposed algorithm on processing speed calibration algorithm is about $40 \%$ increase compared to the cycle.

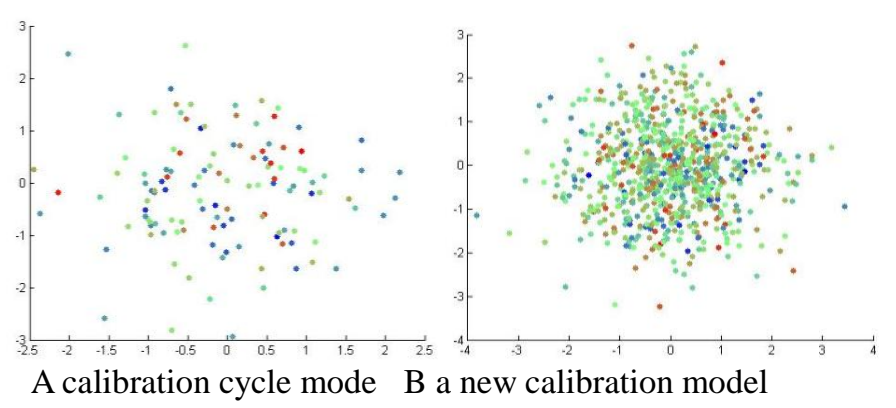

Fig. 8 error distribution

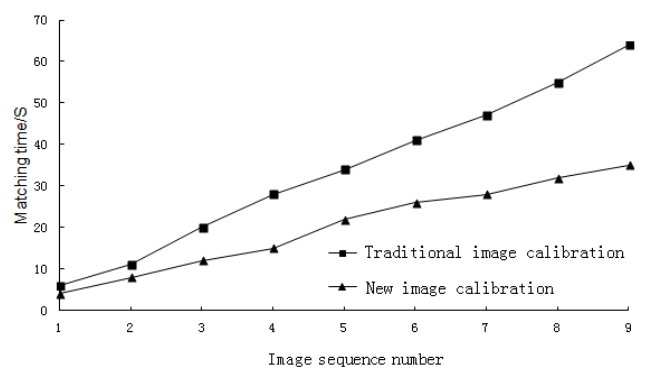

Fig. 9 image matching of time

\section{Conclusion}

In this paper, a camera calibration method based on directional point, and test under the software matlab are proposed, We can get the following conclusions:

(1)Compared checker-board calibration methods, the proposed calibration method based on directional point has the advantages of small amount of calculation of high efficiency, and can satisfy the industrial measurement accuracy. 
(2)In view of the image of 9 different calibration error, two different calibration mode comparative analysis. The calculation results show that the new method on camera calibration parameters to obtain is higher than the accuracy of the calibration cycle mode.

(3)Target matching of time show that this calibration method is more efficient compared with the circular calibration plate calibration method.

\section{Acknowledgements}

This paper is supported by the Natural Science Foundations of Zhejiang Province of China (the Project No. LY13E050026, and Project No.LY14E050025).

\section{References}

[1] WANG L,DUAN F,LU K, An adaptively weighted algorithm for camera calibration algorithm[J]. Pattern Recognition, 2010,43(3):1180-1187.

[2] LIU T,LI Tengfei. Reaserch of the improvement of Zhang's camera calibration method[J]. Optical Technique, 2014,40(5):565-570.

[3] SHAWASH J. SELVIAH D R. Real-time nonlinear parameter estimation using the levenberg marquardt algorith on field programmable gate arrays[J]. IEEE Transactions on Industrial Electronics,2013,60(1):170-176.

[4] RAHMAN T,KROUGLICOF N.An efficient camera calibration technique offering robustness and accuracy over a wide range of lens distortion[J].IEEE Transactions on Image Processing, 2012,21(2):115-122.

[5] MEHRDAD Shahabi, Avinash Unnikrishnan, Stephen D Boyles. An outer approximation algorithm for the robust shortest path problem [J].Transportation Research Part E,2013,5(8):55-66.

[6] AMANPREET Singh, Gianetan Singh Sekhon. A New Shortest Path Finding Algorithm for a Maze Solving Robot With Simulator[J]. Inernational Journal of Computer Science and Communication,2011,2(2):445-449. 